\title{
Safety Assessment of High-Dose Narcotic Analgesia for Emergency Department Procedures
}

From the University of Michigan Medical Center, Ann Arbor;* University of Cincinnati Medical Center, Cincinnati, Ohio ; Vanderbilt University Medical Center, Nashville, Tennessee; ${ }^{\ddagger}$ University of Louisville, Louisville, Kentucky; ${ }^{\S}$ Hennepin County Medical Center, Minneapolis, Minnesota;" and Richland Memorial Hospital, Columbia, South Carolina. ${ }^{q}$

Received for publication April 21, 1992. Revision received January 6, 1993. Accepted for publication April 1, 1993.

Funding for this study was provided by a grant from the Key Pharmaceutical Company, Inc, Miami, Florida.

Presented at the Society for Academic Emergency Medicine Annual Meeting in San Diego, May 1989

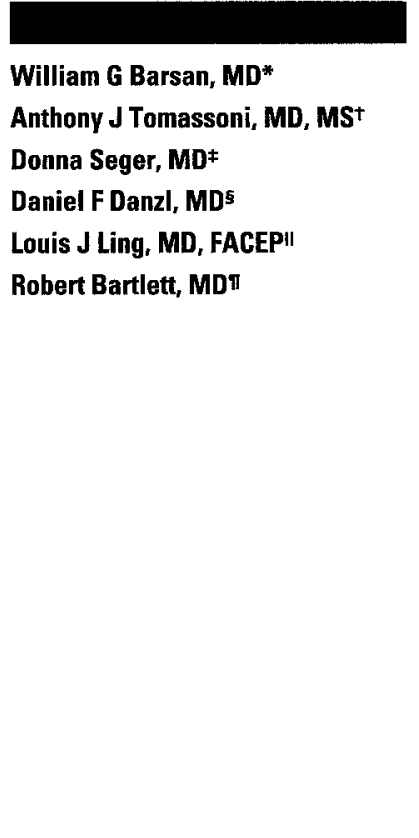

Study objective: To evaluate the safety of high-dose IV narcotics in patients requiring analgesia for painful emergency department procedures.

Design: Prospective multicenter clinical trial.

Setting: Five adult urban EDs.

Methods and measurements: All patients received IV meperidine (1.5 to $3.0 \mathrm{mg} / \mathrm{kg})$ titrated to analgesia followed by a painful procedure. Vital signs and alertness scale were recorded at regular intervals, and patients were observed for four hours. Adverse events were monitored and documented. Comparisons between baseline and postanalgesia intervals were made with a repeated measures ANOVA (Dunnett's test).

Results: Although statistically significant changes in vital signs and alertness scale occurred, they were not clinically significant. Opiate reversal with naloxone was not needed in any patient, and no significant respiratory or circulatory compromise occurred.

Conclusion: This study of 72 patients demonstrates that highdose narcotic analgesia is appropriate, well tolerated, and safe when used in selected patients before painful procedures in the ED. Narcotic antagonists and resuscitation equipment nonetheless should be available to maximize safety.

[Barsan WG, Tomassoni AJ, Seger D, Danzl DF, Ling LJ, Bartlett R: Safety assessment of high-dose narcotic analgesia for emergency department procedures. Ann Emerg Med September 1993;22:1444-1449.] 


\section{INTRODUCTION}

Opiate compounds are used frequently in the emergency department to facilitate the performance of painful procedures by reducing the pain and anxiety experienced by patients. Despite the availability of potent opiate compounds for the relief of pain, studies suggest that substantial numbers of adult and pediatric patients are undermedicated for the degree of pain experienced. ${ }^{1-5}$ Fear of dangerous adverse effects, especially respiratory depression, is an important factor that apparently prevents the use of adequately dosed narcotic analgesia.

No prospective studies have documented the safety of appropriately "high-dose" IV narcotics for procedures in the ED. As a result, patients frequently are given subtherapeutic doses of narcotics for painful procedures, and analgesia is supplemented by "brutane" (ie, insufficient analgesia followed by the necessarily aggressive performance of a procedure). In a retrospective review of 2,000 pediatric cases of facial trauma in which the patients were sedated with IV fentanyl, Billmire et al ${ }^{6}$ reported only thiree cases of apnea requiring antagonist reversal. They concluded that fentanyl is a safe and useful adjunct to the treatment of pediatric facial trauma. Similarly, Chudnofsky et al 7 retrospectively reviewed 841 cases in which fentanyl was used without serious complication in an adult population. No patient required hospitalization for complications in either study.

Intersubject variability in opiate requirements must be recognized. Patient weight is only one of many variables that influence opiate requirements. Older patients demonstrate increased responses to therapeutic doses of meperidine, thought to be the result of decreased protein binding and increased plasma concentrations. ${ }^{8}$ Several variables are known to alter the pain threshold, including sex, alterations in circulation, skin temperature, sweating, $\mathrm{PCO}_{2}$, and anxiety, fear, and other emotions. A given subject may have unequal responses to identical doses of a given narcotic at different times. ${ }^{9}$ Interethnic variability in doses of meperidine required to produce analgesia also have been described. ${ }^{10}$

We conducted a prospective study in patients receiving aggressive doses of IV meperidine in the ED for painful procedures to evaluate the effects on vital signs and. alertness and to document adverse outcomes.

MATERIALS AND METHODS

All patients were participants in a multicenter study comparing two opiate antagonists with placebo in reversing the effects of IV narcotics given to obtain analgesia for time-limited painful procedures. ${ }^{11}$ Seventy patients who received high-dose meperidine followed by placebo (normal saline IV) for narcotic reversal were the subjects of this study.

All patients were between 18 and 65 years old and had conditions for which opiate analgesia was indicated (ie, performance of a painful procedure), including joint reduction, incision and drainage of an abscess, or dilatation and curettage for an incomplete abortion. Informed consent was obtained from all patients before entry in the study. This study was approved by the institutional review boards of all participating institutions.

Patients with a history of allergy to opiates or narcotic antagonists and all pregnant or lactating women were excluded. Other exclusions were recent history of substance abuse, recent ingestion of sedating drugs including opiates (within four half-lives), psychiatric or neurologic impairment, baseline alertness scale score of 19 or less, head injury, and active liver or cardiorespiratory disease. Patients with severe chronic obstructive pulmonary disease or congestive heart failure were specifically excluded as were patients on clonidine, $\alpha$-methyldopa, or guanabenz for hypertension. Patients who were likely to require opiates for ongoing pain control were excluded also.

A history and physical examination were performed on all patients before entry. Peripheral IV access was secured. Baseline vital signs and alertness scale then were determined (Table 1). Opiate analgesia was obtained with IV meperidine administered over 15 minutes to a dose of 1.5 to $3.0 \mathrm{mg} / \mathrm{kg}$, not to exceed $300 \mathrm{mg}$. Meperidine was administered in bouses of 25 to $50 \mathrm{mg}$ with incremental titration until there was adequate analgesia and sedation based on the physicians' clinical assessment. Vital signs (temperature, pulse, respirations, and blood pressure) and alertness scale were repeated. The painful procedure was performed and completed within a 45-minute period. After the procedure, each patient received $1.0 \mathrm{~mL}$ IV normal saline as placebo based on preassigned random codes. Vital signs and alertness scales then were repeated every 15 minutes for one hour and every 30 minutes for the subsequent three hours. Patient follow-up was obtained in 24 to 72 hours.

The alertness scale used was adapted from previously designed and tested scales. It consists of four parts (Table 1). The eye opening and speech scores were adapted from the Glasgow Coma Scale. Level of consciousness was adapted from the Head Injury Watch Sheet, and the commands section was adapted from the Edinburgh-2 Coma Scale. Each of the categories in level of consciousness, eye opening, and speech sections of the scale was 
assigned two points to allow for gradation of response. For example, a patient who responded to conversational tones of voice would score five points, whereas a patient who responded solely to shouting would score only four points.

Values obtained for respiratory rate, systolic and diastolic blood pressure, pulse rate, temperature, and alertness scale after the administration of narcotic analgesia were compared with baseline values using a repeated measures ANOVA (Dunnett's test). All variables were tested to ascertain if their distributions could be considered normally distributed for ANOVA calculations. Only the commands section of the alertness scale was determined to be significantly non-normal, acting essentially as a constant in the total alertness score. When the alertness scale is adjusted by deleting the commands section, the results are almost identical. $\alpha$ was set at .05; therefore, values of $P<.05$ were considered statistically significant.

\section{RESULTS}

Seventy patients, 39 men and 31 women, ranging in age from 18 to 63 years were enrolled in this study. The mean age was 31.1 years. The mean dose of meperidine given to these patients was $173 \mathrm{mg}$ ( 75 to $310 \mathrm{mg}$ ), corresponding to a mean dose of $2.5 \mathrm{mg} / \mathrm{kg}$. The mean time between analgesia and completion of the painful procedure was 24 minutes. No procedure lasted longer than one hour. Diagnoses of the 209 patients admitted to all arms of the study consisted of soft tissue abscess $(72 \%)$, dislocated joints $(12 \%)$, incomplete abortions $(8 \%)$, and other $(8 \%)$

Table 1.

Alertness scale

\begin{tabular}{cl}
\hline Level of Consciousness & \\
$6-7$ & Awake \\
$4-5$ & Awakens to voice \\
$2-3$ & Awakens to shaking \\
$0-1$ & Awakens to pain \\
Eye Opening & \\
$6-7$ & Spontaneous \\
$4-5$ & To voice \\
$2-3$ & To pain \\
$0-1$ & None \\
Speech & \\
$6-7$ & Clear, spontaneous, appropriate \\
$4-5$ & Clear, appropriate, not spontaneous \\
$2-3$ & Inappropriate but can say one word \\
$0-1$ & Incomprehensible, garbled, slurred \\
Commands (open/close hands; open/close eyes) \\
4 & Both correct \\
2 & One correct \\
0 & Both incorrect \\
& \\
\hline
\end{tabular}

including painful joint injury, burns, lacerations, and thrombosed hemorrhoids.

Thirteen adverse reactions occurred among patients in the placebo arm of the study (Table 2). No patients required naloxone reversal for severe respiratory depression or other indication. No respiratory or cardiac arrests occurred, and no patients required ventilatory assistance, intubation, or CPR. Nausea and vomiting were occasionally noted. Follow-up visits in 24 to 72 hours revealed no significant late side effects.

Respiratory rate dropped from a mean baseline of $20 \pm 4.2$ breaths per minute to a mean nadir of $17 \pm 3.3$ breaths per minute at 15 minutes after the termination of the procedure $(P<.05)$. The lowest respiratory rate at any time in any patient was ten, and no patient required ventilatory assistance or reversal with a narcotic antagonist. The four-hour postprocedure respiratory rate was $18 \pm 3$ breaths per minute $(P<.05)$.

The mean nadir systolic blood pressure (after meperidine) occurred at one and two hours after the termination of the painful procedure and was $123 \pm 14 \mathrm{~mm} \mathrm{Hg}$ and $123 \pm 14 \mathrm{~mm} \mathrm{Hg}(P<.05)$, respectively. The greatest individual drop in systolic blood pressure compared with the baseline measurement was $56 \mathrm{~mm} \mathrm{Hg}$, from 180 to $124 \mathrm{~mm} \mathrm{Hg}$ one hour after the painful procedure. The lowest recorded systolic pressure was $88 \mathrm{~mm} \mathrm{Hg}$ in a patient whose initial reading was $90 \mathrm{~mm} \mathrm{Hg}$. The fourhour postprocedure mean systolic blood pressure was $125 \mathrm{~mm} \mathrm{Hg}$ for comparison with the baseline value of $131 \mathrm{~mm} \mathrm{Hg}$ (not statistically significant).

The baseline mean diastolic blood pressure was $82 \pm 13 \mathrm{~mm} \mathrm{Hg}$, and the mean nadir diastolic blood pressure (after meperidine) was $77 \pm 11 \mathrm{~mm} \mathrm{Hg}(P<.05)$ 15 minutes after the procedure and $77 \pm 12 \mathrm{~mm} \mathrm{Hg}$ (not statistically significant at the $P<.05$ level) at 30 minutes after completion of the painful procedure. At four hours

Table 2.

Type and frequency of complications encountered with the use of high-dose meperidine

\begin{tabular}{lc} 
Adverse Reaction & Frequency \\
\hline Dizziness & 1 \\
Hypotension & 1 \\
Nausea & 3 \\
Vomiting & 6 \\
Arrhythmia & 1 \\
Other*+ & 1 \\
${ }^{*}$ Includes palpitations, sweating, tachycardia, tinnitus paresthesia, tremor, vasodilation, or \\
vertigo. \\
${ }^{+}$No chest pain, paranoia, dyspepsia, chills, or nervousness were noted.
\end{tabular}


after the procedure, the mean diastolic blood pressure measured $77 \pm 12 \mathrm{~mm} \mathrm{Hg}$ (not statistically different from control).

Mean pulse rate before administration of meperidine was $83 \pm 16$. Mean nadir pulse rates occurred at both 120 and 180 minutes after the end of the procedure and were identical at $73 \pm 13(P<.05)$. After meperidine, the lowest measured pulse rate was 41 in a patient whose baseline rate was 45 . No patient required fluids or drug treatment for hypotension or bradycardia. At four hours after the procedure, the mean pulse rate was $74 \pm 13(P<.05)$.

A clinically insignificant decrease in body temperature (less than $1.0 \mathrm{C}$ ) was noted. This is consistent with the established knowledge that narcotic administration may influence temperature in human beings. ${ }^{12}$

All patients had a baseline alertness score of 25 of a possible 25 points, except for one who began with a baseline score of 24. After administration of meperidine, patients were notably sedated; however, the lowest level of alertness in any patient was 13 of 25 . Mean nadir alertness score was $19 \pm 2.7$ points $(P<.05)$ immediately after analgesia. The mean alertness score remained at its nadir until the end of the procedure and improved steadily thereafter. The mean alertness scale score at four hours was $24 \pm 2.0(P<.05)$. All patients were discharged after the four-hour observation without any reported complications after discharge. Only the commands section of the alertness scale showed significant non-normality, acting essentially as a constant.

\section{DISCUSSION}

Parenteral narcotic analgesia has been the standard for providing pre- and postoperative pain relief in the hospitalized patient since the invention of the hollow needle and syringe in 1853. Meperidine was synthesized first in 1939 and initially enjoyed popularity as an anesthetic in conjunction with nitrous oxide..$^{13} \mathrm{It}$ is approximately oneeighth to one-tenth as potent as morphine. Meperidine often is selected because of its intermediate duration of action, oral availability, and less resultant spasmogenicity when compared with other opiates (excluding the agonistantagonist class of opiates). It is suitable for the performance of painful procedures and postprocedure analgesia, with the exception of bronchoscopy where its relative lack of antitussive effect makes it less desirable than other opiates. Jaffe and Martin ${ }^{12}$ stated that physicians often prescribe doses of meperidine that are too low or too infrequent, thereby causing needless suffering.
IM use of meperidine results in variable pain control within a given subject when studied at regular dosing intervals. This effect is due to two variables: unpredictable absorption from the IM site and large interpatient variability between blood meperidine concentration and analgesic effect. In addition, the dose-response curve for meperidine is steep, with small increases in blood meperidine concentration producing a dramatic effect on analgesia. Elimination of the absorption step by IV administration and titration of drug levels in response to pain rather than strict adherence to body weight dosing may be expected to yield improved analgesia. ${ }^{14,15}$

Physician and patient attitudes regarding assessment and expression of pain along with fear of opioid dependence are often based on fallacious reasoning. These factors must be overcome to provide effective pain management. Improved understanding of narcotic dependency, tolerance, patient behavior, prescribing errors, analgesic equivalencies, and effect of route of administration should facilitate effective pain management. Frequent reinforcement of the hazards associated with narcotic administration and too little teaching about proper use of these agents, potential misunderstanding of equianalgesic doses of different narcotics, and apprehension on the part of nurses and other staff may further contribute to the high incidence of oligoanalgesia, especially in the litigious climate surrounding medicine today. 3,16,17

In a study with some parallels to ours, a clinical neurologic and behavioral scoring system developed for assessment of pain in infants facilitated use of narcotic analgesia in this group historically undertreated for pain. No significant differences were noted between those receiving $3 \mu \mathrm{g} / \mathrm{kg}$ fentanyl and controls with respect to transcutaneous $\mathrm{PO}_{2}$ or $\mathrm{PCO}_{2}$, oxygen saturation, blood pressure, heart rate, or temperature. A faster respiratory rate was noted in controls. This was attributed to pain. ${ }^{18}$

Although statistically significant differences in vital signs and alertness scale were evident, there were no episodes of airway compromise, respiratory arrest, or hemodynamic instability. The modest declines in fourhour blood pressure values, pulse rates, and respiratory rates compared with baseline might reflect a decrease in the level of the patients' pain and apprehension after the correction of their underlying medical problem. Maximal respiratory depression occurred at 15 minutes after the painful procedure in most patients, a point when narcotic blood level is still high and pain and anxiety have been decreased because of correction of the underlying medical problem. This finding supports the premise that extended observation in the ED after narcotic analgesia may not be 
warranted solely because of fear of respiratory depression. No instance of respiratory depression or rebound adverse effect after discharge was reported by patients on questioning at follow-up. Regarding other vital signs, systolic blood pressure reached a nadir at 120 minutes, diastolic blood pressure at 60 minutes, and pulse at both 120 and 180 minutes, with no patient becoming hemodynamically unstable or requiring resuscitation. The individual nadir respiratory rate of ten breaths per minute (obtained at termination of the painful procedure) further supports the contention that observation periods in the ED may be shortened even with high-dose meperidine administration.

The placebo effect was not controlled for in this subpopulation from a larger study. Similarly, the effect of placebo in this control arm of a larger study of 209 patients (in place of nalmefene) is an extraneous variable whose effect, although presumed to be small, is unknown. Two complications were noted in the larger study. Neither patient belonged to the study arm from which our data were derived. One patient in the larger study had potentially dangerous respiratory depression, received unblinded naloxone, and was not included in any study data. A second patient received naloxone for an inadvertent overdose of $200 \mathrm{mg}$ of meperidine fast IV push and was likewise not included in the study data.

Where used episodically in the ED for the performance of a painful procedure, narcotic abuse potential and liability are not likely to be of concern except perhaps in the reformed addict. Safety dictates that absolute and relative contraindications to the use of meperidine must be recognized (Figure). Narcotic administration may cause nausea and vomiting, increasing the risk of aspiration of gastric contents in patients who have eaten shortly before sedation. Increased potential for adverse effects must be recognized when narcotic adjuncts such as benzodiazepines are used in conjunction with narcotics. Frequent evaluation of vital signs and alertness coupled with ECG and oxygen saturation monitors should be standard wherever conscious sedation is used to improve the margin of safety for patients receiving large doses of narcotics and other sedative medications. A narcotic antagonist such as naloxone and standard advanced cardiac life support resuscitation equipment should be available along with personnel skilled in the use of these materials for patient protection in the event of inadvertent overdose.

Clinically unrecognized hypoxemia has been documented during conscious sedation in patients monitored by pulse oximeter. Properly set audible alarms on monitoring equipment are essential to prevent unrecognized respiratory or circulatory collapse in unsupervised patients after the procedure, when reduction in the magnitude of painful stimuli may depress their mental status and vital signs. Unfortunately, at the time of this study, pulse oximetry was not widely available in the ED. Capnography by nasal cannula also is undergoing study in this setting, allowing the monitoring of both end-tidal $\mathrm{PCO}_{2}$ and respiratory patterns. Although capnometers are not generally available in EDs at this time, improvements in clinical care coupled with technologic advancement may virtually mandate their future use during conscious sedation. 19

The established practice of observing patients for two to four hours to ensure safe discharge from the ED after the administration of potentially sedating doses of narcotics may be a deterrent to the administration of narcotic analgesia in a busy ED setting. Fear of rebound respiratory depression may be a contributing factor and may contribute to the avoidance of adequate dose analgesia. With additional study to demonstrate the safety of conscious sedation using short-acting drugs in adequate therapeutic doses, observation time might be decreased. Short-acting narcotics such as fentanyl (another phenylpiperadine congener of meperidine), sufentanil, or alfentanil may soon become the standard of care for conscious sedation in outpatient settings. Alternatively, the introduction of a narcotic antagonist similar to nalmefene may provide the impetus for physicians to use adequate dose analgesia for the performance of painful procedures in the outpatient setting with the assurance that a reliable longacting narcotic reversal agent can provide. ${ }^{11}$ Our work suggests that a two-hour interval may be sufficient observation time for many patients. Patients may be discharged when they are awake and alert without clinical signs of sedation or respiratory depression. It is our practice to

\section{Figure.}

Absolute and relative contraindications to meperidine

Hypersensitivity

Head injury and increased intracranial pressure

Asthma, chronic obstructive pulmonary disease, or pre-existing respiratory depression

Congestive heart failure or ischemic cardiac disease

Pre-existing hypotension

Monoamine oxidase inhibitors

Cautions:

Pregnancy

Seizure disorder

History of tachyarrhythmias

Conditions causing delayed drug metabolism and clearance

(especially liver disease) 
NARCOTIC ANALGESIA

Barsan et al

discharge patients to the care of a family member or similar caretaker with instructions to avoid driving and other potential hazards, including the consumption of alcohol and other depressant drugs for a period equal to several half-lives of the narcotic administered.

\section{CONCLUSION}

Although the administration of large doses of meperidine produced statistically significant changes in respiratory rate, blood pressure, and pulse rate, these changes were not clinically significant. Changes in alertness score reflected analgesia and sedation sufficient to allow the performance of a painful procedure but reverted back to baseline thereafter without clinically significant sequelae at any time. We conclude that appropriately high doses of meperidine are well tolerated and safe when given to patients without significant cardiorespiratory disorders or other contraindications before painful procedures in the ED.

\section{REFERENCES}

1. Wilson JE, Pendletor JM: Oligoanalgesia in the emergency department. Am J Emerg Med 1989;7:620-623.

2. Marks RM, Sachar EJ: Undertreatment of medical inpatients with narcotic analgesics Ann intern Med 1973;78:173-181.

3. Sriwatanakul K, Weis OF, Aloza JL, et al: Analysis of narcotic analgesic usage in the treatment of postoperative pain. JAMA 1983;250:926-929.

4. Reichl M, Bodiwala GG: Use of analgesia in severe pain in the accident and emergency department. Arch Emerg Med 1987;4:25-31.

5. Selbst SM, Clark M: Analgesic use in the emergency department. Ann Emerg Mod 1990;19:1010-1013.

6. Billmire DA, Neal HW, Gregory PO: Use of IV fentanyl in the outpatient treatment of pediatric facial trauma. J Trauma 1985;25:1079-1080.

7. Chudnofsky CR, Wright SW, Dronen SC, et al: The safety of fentanyl use in the emergency department. Ann Emerg Med 1989:18:635-639.

8. Mather LE, Tucker GT, Pflug AE, et al: Meperidine kinetics in man. Clin Pharmacol Ther 1975:17:21-30.

9. Swinyard EA: Analgesics and antipyretics, in Gennaro AR (ed): Remington's Pharmaceutical Sciences, ed 18. Easton, Pennsylvania, Mack Publishing Co, 1990, p 1097-1106.

10. Houghton IT, Aun CS, Gin T, et al: Inter-ethnic differences in post operative pethidine requirements. Anaesth intensive Care 1992:20:52-55.

11. Barsan WG, Seger D, Danzl DF, et al: Duration of antagonistic effects of nalmefene and naloxone in opiate-induced sedation for emergency department procedures. Am J Emerg Med 1989:7:155-161.

12. Jaffe $\mathrm{JH}$, Martin WR: Opiate analgesics and antagonists, in Goodman LS, Gilman AG, Gilman A (ed's): The Pharmacological Basis of Therapeutics, ed 6. New York, Macmillan, 1980, p 494-531.

13. Bailey PL, Stanley TH: Pharmacology of Intravenous Narcotic Anesthetics, in Miller RD (ed): Anesthesia, ed 2. New York, Churchill-Livingstone, 1986.

14. Austin KL, Stapleton JV, Mather LE: Multiple intramuscular injections: A major source of variability in analgesic response to meperidine. Pain 1980;8:47-62.

15. Austin KL, Stapleton JV, Mather LE: Relationship between blood meperidine concentrations and analgesic response: A preliminary report. Anesthesiology 1980;53:460-466

16. Rogers AG: Analgesics: The physician's partner in effective pain management. Va Med 0 1989:116:164-170
17. Yaster $M$, Deshpande JK, Maxwell $L G$ : The pharmacologic management of pain in children Compr Ther 1989;15:14-26.

18. Barrier G, Attia J, Mayer MN, et al: Measurement of post op pain and narcotic administration in infants using a new clinical scoring system. Intensive Care Med 1989;15:\$37-S39.

19. Wright $S W$ : Conscious sedation in the emergency department: The value of capnography and pulse oximetry. Ann Emerg Med 1992;21:551-555.

\section{Address for reprints:}

Anthony J Tomassoni, MD, MS

Department of Emergency Medicine

University of Cincinnati Center for Emergency Care

234 Goodman Street

Cincinnati, Ohio 45267-0769 